\title{
Approches théoriques de la réécriture
}

\author{
Christian Milat \\ Université d'Ottawa
}

La réécriture est, sur le plan théorique, un domaine assez complexe, voire confus : les concepts s'y succèdent, les mêmes termes pouvant parfois posséder des acceptions tout à fait différentes. Mon propos n'est donc pas ici d'enchérir au risque d'ajouter à la confusion, mais seulement de tenter, au risque de la simplification, d'aller à l'essentiel en étant aussi clair que possible.

Je commencerai par recourir aux dictionnaires et notamment au Trésor de la langue française. Même si les articles concernés ne sont pas très éclairants, il reste qu'ils sont néanmoins intéressants dans la mesure où, justement, ils sont symptomatiques de la difficulté à saisir ces notions. Deux 
acceptions ne concernent pas l'objet, éminemment littéraire, qui nous intéresse ici : "récrire » ou "réécrire»- les deux formes existent - comme "écrire une nouvelle fois (une deuxième, une troisième... fois) un texte à quelqu'un » - le préfixe ré- ne marquant que la répétition - et, au sens figuré et dans le registre familier, "réinventer, donner une nouvelle vision de quelque chose ». Plus près de nos préoccupations, « récrire» ou "réécrire», signifie également «donner une nouvelle version d'un texte déjà écrit». Le Petit Robert, lui, propose comme définition "écrire ou rédiger de nouveau», notamment pour "améliorer la forme» (p. 2200), et dans ce cas, il donne comme synonyme l'anglicisme « rewriter ». Après le verbe, allons voir le nom. $\mathrm{Si}$ « récriture » est absent des deux dictionnaires, le mot « réécriture », lui, y figure. Le Trésor de la langue française se contente de le définir comme «l'action, le fait de réécrire» et Le Petit Robert, lui, indique aussi tout naturellement : «action de réécrire un texte pour en améliorer la forme ou pour l'adapter à d'autres textes, à certains lecteurs », et donne comme synonyme l'anglicisme « rewriting » (p. 2207). Nous n'avons pas beaucoup progressé...

\section{La réécriture génétique}

Néanmoins, nous possédons une piste de départ. Que doit-on entendre par «donner une nouvelle version d'un texte déjà écrit » ou par « écrire ou rédiger de nouveau »? Je vous propose de délaisser l'acception relative au domaine de l'édition, à la rédactologie, à laquelle les anglicismes font référence, pour nous concentrer sur les pratiques littéraires. 
L'une d'entre elles comprend le travail que l'écrivain réalise sur le ou les premiers états de son manuscrit, car le texte définitif d'une œuvre littéraire ne correspond pas, à de très rares exceptions près, au premier jet, mais est le résultat de multiples corrections et révisions. Ce type de réécriture est au principe de la génétique textuelle (qui analyse les différents avant-textes, les différents manuscrits d'une œuvre, qui les classe, qui les déchiffre, qui en relève les variantes), cette génétique textuelle débouchant sur la critique génétique (laquelle interprète les résultats obtenus à la suite de ce travail).

Tout à fait différent de cette réécriture génétique et correspondant au sujet du présent colloque apparaît un autre type de réécriture, qui implique cette fois la claire volonté d'un écrivain de réécrire des textes déjà publiés, qu'il s'agisse de l'œuvre d'un autre auteur ou de l'une de ses propres œuvres.

\section{De nombreux réécrivains}

De nombreux écrivains sont ainsi des réécrivains : ils ont composé leurs œuvres à partir d'œuvres antérieures.

Je ne vais pas vous présenter l'histoire, même abrégée, de cette réécriture. Je me contenterai de vous donner seulement quelques balises, de façon à illustrer le fait que le procédé s'étale sur une longue période.

Ainsi, Le Décaméron de Boccace a été suivi, en 1462, soit un peu plus d'un siècle après sa parution, par le recueil des Cent nouvelles nouvelles puis, en 1559, soit encore à peu un siècle après, par celui de Marguerite de Navarre, L'Heptaméron. 
L'esthétique classique et l'imitation des Anciens qu'elle implique notamment donnent bien évidemment une large place à la réécriture: pensons seulement à l'Iphigénie à Aulis d'Euripide et à l'Iphigénie de Racine ou aux Fables d'Ésope et à celles de La Fontaine. Beaucoup plus près de nous, citons, à titre d'exemples, Les Trois Médecins, la réécriture, publiée par Martin Winckler en 2004, du roman d'Alexandre Dumas, ou le vingt-etunième roman d'Amélie Nothomb, paru en 2012 et intitulé Barbe bleue.

Mais je voudrais évoquer plus en détail un des cas les plus illustratifs de la réécriture. Il trouve son origine dans A Cruising Voyage Round the World, journal publié en 1712 par un corsaire anglais, le capitaine Woodes Rogers. Dans ce récit autobiographique, Rogers raconte comment, en 1709, a été secouru un certain Alexandre Selkirk, un marin écossais qui, à sa demande, a été débarqué, quatre ans auparavant, sur une île située à $600 \mathrm{~km}$ au large du Chili par le commandant avec qui il ne s'entendait pas. Ce récit sera réécrit par l'écrivain anglais Daniel Defoe, qui en fera un roman, publié en 1719, La Vie et les aventures étranges et surprenantes de Robinson Crusoé de York, marin. Des transformations sont apportées par Defoe par rapport au texte de Woodes Rogers : par exemple, l'action se passe en 1659 (au lieu de 1709) et, naufragé, Robinson se retrouve cette fois involontairement seul sur une île de l'océan Atlantique, et non plus du Pacifique. Au début du XXe siècle, Robinson Crusoé fournit la trame d'un poème, «Images à Crusoé », du recueil Éloges que Saint-John Perse publie en 1904 et, en 1926, il est à l'origine du roman de Jean Giraudoux, Suzanne et le Pacifique, où, contrairement à ce qui se passe chez Defoe, c'est le personnage de Vendredi qui tient le rôle principal. Cette transvalorisation sera conservée en 1967 dans 
le roman de Michel Tournier, Vendredi ou les limbes $d u$ Pacifique. Quatre ans plus tard, Tournier lui-même, pour le destiner aux jeunes, réécrit son propre roman, intitulé alors Vendredi ou la vie sauvage, et, en 1978, sous la forme d'une nouvelle, "La fin de Robinson Crusoé », qui fait partie du recueil intitulé Le Coq de bruyère, il apporte un épilogue à son roman. Robinson et Vendredi sont retournés en Angleterre. Vendredi ayant disparu, Robinson quitte Londres pour aller à sa recherche, mais il ne retrouve ni Vendredi ni son île, disparue comme est disparue également sa jeunesse. Finalement, en 2012, c'est Patrick Chamoiseau qui publie une nouvelle réécriture, toute différente, du roman de Defoe : L'Empreinte à Crusoé.

J'ai emprunté ces exemples à la littérature française, mais la réécriture est présente dans toutes les littératures, un des textes emblématiques du procédé étant bien évidemment Ulysse, la réécriture réalisée par Joyce faisant du reste l'objet d'une communication. Et, au Québec, pensons par exemple, au conte de Jacques Ferron, intitulé « Le petit chaperon rouge».

\section{Intertextualités}

La réécriture consciente d'un texte $A$ dans un texte $B$ peut laisser présager la présence, dans le second texte, de traces provenant du premier. D’où la relation possible entre réécriture et intertextualité.

C'est Julia Kristeva (1967) qui, la première, parle d'intertextualité : «l'écriture [apparaît] à la fois comme subjectivité et comme communicativité ou, pour mieux dire, 
comme intertextualité ». Cette première théorie de l'intertextualité s'appuie sur le dialogisme tel qu'il est défini en 1929 par Mikhaill Bakhtine (1970), dont les textes sont diffusés en France à partir du milieu des années 1960, notamment grâce à Tzvetan Todorov (1965). Bakhtine développe une idée qui est d'ailleurs exprimée dès 1897 par Mallarmé dans Crise de vers : «plus ou moins, tous les livres contiennent la fusion de quelques redites comptées» (p.367). Pour Bakhtine, le dialogisme est inhérent à l'écriture. En effet, tout énoncé, par sa dimension linguistique, renvoie à d'autres énoncés, à d'autres textes, car tout mot («le mot plein ») a déjà servi : «Le mot n'est pas une chose, écrit Bakhtine (p.48), mais le milieu toujours dynamique, toujours changeant dans lequel s'effectue l'échange dialogique ». À travers la voix d'un écrivain, tout particulièrement d'un romancier - puisque le roman est le genre polyphonique par excellence - , c'est donc un nombre infini d'autres voix qui s'expriment.

Développée en 1969 par Kristeva dans Sèméiôtikè — «Tout texte se construit comme une mosaïque de citations, tout texte est absorption et transformation d'un autre texte.» (p.146) -, cette conception de l'intertextualité est celle que Philippe Sollers présente dans un chapitre de Théorie d'ensemble: "Tout texte se situe à la jonction de plusieurs textes dont il est à la fois la relecture, l'accentuation, la condensation, le déplacement et la profondeur. » (p. 75). C'est également elle qui est reprise en 1973 par Roland Barthes :

Tout texte est un intertexte; d'autres textes sont présents en lui, à des niveaux variables, sous des formes plus ou moins reconnaissables [...]. Passent dans le texte, redistribués en lui, des morceaux de codes, des formules, des modèles rythmiques, des fragments de langage sociaux, etc., car il y a toujours du 
langage avant le texte et autour de lui. [...] L'intertexte est un champ général de formules anonymes, dont l'origine est rarement repérable, de citations inconscientes ou automatiques, données sans guillemets.

Ce concept théorique était peu productif, puisque tout texte était considéré comme un intertexte. Laurent Jenny va le rendre plus opératif en lui donnant un autre contenu, plus restreint : l'intertextualité désigne « le travail de transformation et d'assimilation de plusieurs textes opéré par un texte centreur qui garde le leadership du sens » (p. 262). L'intertextualité n'est plus un fait linguistique qui existe nécessairement et qui s'applique à tous les textes indistinctement; elle est désormais le résultat du travail conscient réalisé par un écrivain sur un texte préexistant.

Ainsi formulée, écrit Marc Angenot en 1983, l'intertextualité consiste "à faire du neuf avec du vieux, et par exemple à appeler analyse intertextuelle une bien traditionnelle critique philologique des sources et des influences littéraires » (p. 125-126), critique qui, faisant la part belle aux auteurs, est alors considérée comme une abomination.

En fait, Angenot semble avoir oublié une précision apportée par Michael Riffaterre : l'intertextualité ne demande pas de " prouver le contact entre l'auteur et ses prédécesseurs. Il suffit, pour qu'il y ait intertexte, que le lecteur fasse nécessairement le rapprochement entre l'auteur et ses prédécesseurs » (1979a, p. 131). Autrement dit, c'est à chaque lecteur qu'il incombe, selon la nature de sa culture - selon la nature de son encyclopédie, dirait Umberto Eco — , d'identifier l'intertexte d'un texte, notamment en repérant et en interprétant ces microstructures problématiques, parce qu'en dissonance avec le reste du texte, que sont les agrammaticalités 
sémantiques, syntaxiques ou morphologiques contenues dans un texte. Dans La Production du texte (1979), Riffaterre donne un exemple d'agrammaticalité tiré d'un texte de Baudelaire («Les tentations, ou Éros, Plutus ou la gloire», dans Petits Poëmes en prose. Les Paradis artificiels) : «Il y avait [...] de vieilles mères portant des avortons accrochés à leurs mamelles exténuées »(1979b, p.56). Selon Riffaterre, il n'y a pas de « chaîne associative » (métonymique, métaphorique ou autre) permettant de dériver «accroché du mot avorton» (p. 56). Autrement dit, la dissonance ou l'« agrammaticalité » des mots manifeste la présence d'un intertexte. Dans ce cas, ce sera la phrase stéréotypée des mères portant des nourrissons suspendus à leurs seins (p. 57). La phrase de Baudelaire se construit alors, par introduction de connotations négatives, en opposition à cette phrase déjà existante : le mot «mères », qui dans son sens positif se relie à la jeunesse, devient «vieilles mères »; le mot «nourrissons» se transforme en «avortons» et le verbe « suspendre » est remplacé par « accrocher », verbe qui, selon Riffaterre, comporte des connotations négatives. Dans « La trace de l'intertexte», Riffaterre confirme que, selon lui, l'intertextualité et, partant, le sens d'un texte ne sont plus des produits de l'écriture, mais des effets de la lecture, toujours aléatoire.

Jusque-là, l'intertextualité s'intéresse essentiellement aux intertextes littéraires. En 1987, Marc Eigeldinger propose de ne pas

limiter la notion d'intertextualité à la seule littérature, mais de l'étendre aux divers domaines de la culture. Elle peut être liée à l'émergence d'un autre langage à l'intérieur du langage littéraire ; par exemple celui des beaux-arts et de la musique, 
celui de la Bible ou de la mythologie, ainsi que celui de la philosophie. (p. 15)

Cette ouverture sera reprise, l'année suivante, par Michel Pierssens, dans un article où il assigne à l'épistémocritique le rôle de donner une réponse à cette question "que sait un texte? » dans la revue Spirale : il s'agit d'analyser la présence et le fonctionnement, dans les textes littéraires, de savoirs non littéraires (philosophiques, scientifiques, etc.).

D’ordinaire, les théories de l'intertextualité font référence à un intertexte qui est antérieur au texte qui l'accueille. Cependant, il est possible d'envisager un intertexte qui lui est postérieur. Cette possibilité, qui met de l'avant le rôle du lecteur dans la détermination de l'intertexte, est évoquée par Roland Barthes :

Lisant un texte rapporté par Stendhal (mais qui n'est pas de lui) j'y retrouve Proust par un détail minuscule. [...] Ailleurs, mais de la même façon, dans Flaubert, ce sont les pommiers normands en fleurs que je lis à partir de Proust. [...] Je comprends que l'œuvre de Proust est, du moins pour moi, l'œuvre de référence, la mathésis générale [...]. Et c'est bien cela l'inter-texte : l'impossibilité de vivre hors du texte infini - que ce texte soit Proust, ou le journal quotidien, ou l'écran télévisuel (p. 58-59).

Cette intertextualité inversée est au principe de l'essai publié par Pierre Bayard, Le Plagiat par anticipation.

\section{Réécriture, intertextualité et hypertextualité}

Ayant passé en revue, même rapidement, quelques définitions de l'intertextualité, nous sommes maintenant en situation 
d'examiner dans quelle mesure les théories de l'intertextualité peuvent rendre compte de la réécriture.

Si la réécriture implique, chez l'auteur, l'intention de réécrire, l'intertextualité inversée, propre au lecteur, est bien évidemment à exclure: la réécriture est obligatoirement la réécriture d'un texte antérieur.

Si la réécriture implique également la réécriture de la structure entière ou quasi entière d'un texte, il faut exclure de la réécriture ce que Laurent Jenny appelle «l'intertextualité "faible" » (p. 262), c'est-à-dire « la présence dans un texte d'une simple allusion ou réminiscence, c'est-à-dire chaque fois qu'il y a emprunt d'une unité textuelle abstraite de son contexte et insérée telle quelle dans un nouveau syntagme textuel, à titre d'élément paradigmatique » (p. 262). Doit donc être également exclue de la réécriture l'intertextualité telle que la conçoit notamment Riffaterre, qui s'intéresse aux microstructures, et telle que la définit Gérard Genette, à savoir comme la « relation de coprésence entre deux ou plusieurs textes, c'est-à-dire éidétiquement et le plus souvent, par la présence effective d'un texte dans un autre»(p.8), cette présence pouvant prendre une forme explicite et littérale - on a alors affaire à des citations - , une forme moins explicite - il s'agit de plagiat (l'emprunt est non déclaré, mais il est encore littéral) - , ou une forme encore moins explicite et non littérale, celle que prend l'allusion.

En revanche, la définition que Laurent Jenny donne de l'intertextualité peut correspondre à ce que nous avons circonscrit comme étant la réécriture puisqu'il propose, «de parler d'intertextualité seulement lorsqu'on est en mesure de repérer dans un texte des éléments structurés antérieurement à 
lui, au-delà du lexème, cela s'entend » (p. 262) et qu'il souligne que «toute une mise en scène fictionnelle [...] se trouve [...] empruntée, adaptée, pervertie et contredite par le travail intertextuel» (p. 263) Et Jenny de dresser la liste des «types d'altérations subies par des textes au cours du procès intertextuel » (p. 275).

Cette conception de l'intertextualité/réécriture proposée par Laurent Jenny est en grande part analogue à la définition que, dans Palimpsestes, Gérard Genette donne de sa cinquième relation transtextuelle, l'hypertextualité. Le titre de l'ouvrage, Palimpsestes, est d'ailleurs tout à fait révélateur puisque, venant du grec palimpsestos, qui signifie «qu'on gratte pour écrire de nouveau », palimpseste réfère à un manuscrit sur parchemin d'auteurs anciens que les moines copistes du Moyen Âge ont effacé pour le recouvrir d'un second texte, le texte original ne disparaissant pas complètement: on voit donc, sur un même parchemin, un texte se superposer à un autre, qu'il ne dissimule pas tout à fait, mais qu'il laisse voir par transparence. Conformément à sa métaphore, Genette définit l'hypertextualité comme «toute relation unissant un texte $\mathrm{B}([\ldots]$ hypertexte $)$ à un texte antérieur A ([...] hypotexte) sur lequel il se greffe d'une manière qui n'est pas celle du commentaire » (p. 11-12). Notons que l'hypertexte, selon Genette, est la plupart du temps un texte de fiction : il ne peut en être autrement qu'en cas de traduction ou de réduction. Il apparaît comme «tout texte dérivé d'un texte antérieur par transformation [l'objectif est de (dire la même chose autrement)] [...] ou par [...] imitation [l'objectif est alors de dire autre chose semblablement]» (p. 14). Cette dérivation doit être «à la fois massive (toute une œuvre $\mathrm{B}$ dérivant de toute une œuvre A) et déclarée, d'une manière plus ou moins officielle » (p. 16). 
Cela posé, Genette distingue six pratiques hypertextuelles. Trois se rapportent au procédé de l'imitation (dire autre chose semblablement) :

tout d'abord, dans le cas du régime ludique (il ne s'agit que de divertir), Genette parle de pastiche; c'est le cas des pastiches que Proust a publiés en 1908 et 1909 dans Le Figaro et dont le recueil en volume, refusé par le Mercure de France, par Calmann-Lévy et par Fasquelle, paraîtra finalement en 1919 chez Gallimard sous le titre de Pastiches et mélanges; écrit à la manière de Saint-Simon, de Balzac, de Flaubert, de SainteBeuve, d'Henri de Régnier, des Goncourt, de Michelet, de Faguet et de Renan ${ }^{1}$, chaque texte évoque un des aspects de l'affaire Lemoine, ingénieur qui avait vendu à la De Beers un faux procédé pour fabriquer des diamants.

- ensuite, dans le cas du régime satirique, Genette parle de charge (l'exagération des traits stylistiques provoque le ridicule); le texte d'Umberto Eco, «Esquisse d'un nouveau chat», en constitue un bon exemple; Eco s'y moque de Robbe-Grillet, dont voici un extrait de Dans le labyrinthe :

De la commode à la table il y a six pas : trois pas jusqu'à la cheminée et trois autres ensuite. Il y a cinq pas de la table au coin du lit ; quatre pas du lit à la commode. Le chemin qui va de la commode à la table n'est pas tout à fait rectiligne : il s'incurve légèrement pour passer plus près de la cheminée. Au-dessus de la cheminée il y a une glace, une grande glace rectangulaire fixée au mur. Le pied du lit est situé juste en face. (p. 59)

Et voici la charge produite par Eco :

1 Proust (p. 594) évoque la vertu « exorcisante » du pastiche : «Il faut faire un pastiche volontaire, pour pouvoir après cela redevenir original, ne pas faire toute sa vie du pastiche involontaire. » 
Du coin de la pièce à la table, il y a six pas. De la table au mur du fond, il y a cinq pas. En face de la table, s'ouvre une porte. De la porte au coin dans lequel vous vous trouvez, il y a six pas. Si vous regardez devant vous de sorte que votre regard traverse la pièce en diagonale, en direction du coin opposé, à la hauteur de vos yeux, au moment où vous vous trouvez pelotonné dans le coin, le museau tourné vers la pièce, la queue entortillée dans la tentative d'effleurer en même temps deux de ses murs qui se rencontrent en formant un angle de quatre-vingt-dix degrés, vous verrez alors, à six pas devant vous, une forme cylindrique d'un marron foncé brillant, sillonnée d'une série de fines veinures au milieu desquelles on entrevoit un cœur blanchâtre, avec une écaillure à environ cinq centimètres du sol, qui s'élargit en une circonférence irrégulière, s'approchant d'une forme polygonale imprécise, au diamètre maximum de deux centimètres; elle révèle un fond tirant lui aussi sur le blanc, mais d'un blanc plus pâle que celui des veinures, comme si, pendant plus longtemps et avec plus de facilité, la poussière s'y était déposée, au fil des jours ou des mois, des siècles ou des millénaires. (p. 73)

- enfin, dans le cas du régime sérieux, Genette parle de forgerie (il s'agit de poursuivre ou d'étendre une œuvre littéraire préexistante); les faux poèmes de Rimbaud publiés en 1949 illustrent parfaitement le procédé de la forgerie²

\footnotetext{
2 En juillet 1872, Verlaine et Rimbaud s'enfuient à Londres. Mathilde Mauté, la femme de Verlaine, découvrant des lettres de Rimbaud montrant les relations homosexuelles entre celui-ci et son époux, utilise ces missives pour obtenir le divorce. Afin de les récupérer, Verlaine prétend qu'elles contiennent des vers et des poèmes en prose. Le 19 mai 1949, Maurice Nadeau annonce dans Combat que le manuscrit a été retrouvé par hasard et qu'il paraît le jour même au Mercure de France sous le titre La Chasse spirituelle. Il s'agit d'une plaquette de 34 pages regroupant cinq textes: "Vaudeville», "Vacances païennes », «Édens », «Infirmités » et « Marécages ». Préfacé par Pascal Pia, l'ouvrage met en lumière des parallèles avec certains passages bien connus de l'œuvre rimbaldienne comme. Ce sera André Breton qui, dans une lettre publiée dans Combat, puis dans une autre dans Le Figaro, puis dans son pamphlet " Flagrant délit », affirmera qu'il est impossible que ces textes soient de Rimbaud. De fait, le 2 juillet, le Mercure de France retire l'ouvrage de la
} 


\section{Parodie, travestissement et transposition}

Trois pratiques se rapportent également au procédé de la transformation (dire la même chose autrement) :

- dans le cas du régime ludique, Genette parle de parodie; ce poème tiré d'un recueil anonyme publié en 1694, est un bon exemple de parodie :

La Cigale ayant baisé

Tout l'été

Se trouva bien désolée

Quand Langeron l'eut quittée.

Pas le moindre pauvre amant

Pour soulager son tourment.

Elle alla crier famine

Chez la Grignan sa voisine,

La priant de lui prêter

Un Grignan pour subsister

Jusqu'à la saison nouvelle.

Je vous le rendrai, dit-elle,

Avant qu'il soit quatre mois,

Sans l'avoir mis aux abois.

La Grignan n'est pas prêteuse,

C'est là son moindre défaut.

Lequel est-ce qu'il vous faut?

Dit-elle à cette emprunteuse.

Le chevalier de Grignan,

Dit la triste tourterelle.

Le chevalier, lui dit-elle,

J'en ai besoin maintenant ${ }^{3}$. (p. 231)

vente. Les auteurs de ce faux se dénoncent: il s'agit de deux comédiens, Nicolas Bataille et Mlle Akakia-Viala, dont la mise en scène théâtrale d'Une saison en enfer avait été éreintée par la presse, avaient voulu ridiculiser leurs critiques.

3 La Cigale est Françoise Charlotte de Montalais, comtesse de Marans, et la Grignan, Françoise Marguerite de Sévigné, comtesse de Grignan, fille de Madame de Sévigné. 
- dans le cas du régime satirique, Genette parle de travestissement: sans modifier le sujet, on modifie le style, de noble à vulgaire. C'est par exemple le cas de Paul Scarron, qui travestit l'Énéide dans, précisément, Le Virgile travesti. Voici ainsi ce que Didon, reine de Carthage, dit à Énée, le Troyen :

Tu n'es qu'un sot, tu n'es qu'un fat,

Tu n'es qu'un larron comme un rat [...].

Tu te dis fils de Cythérée :

La chose n'en est assurée

Qu'en tant que grand fils de putain; [...]

Non, je me trompe, c'est un loup

Qui t'engendra d'une panthère;

Aucuns disent une vipère

Qui te conçut d'un léopard;

Les autres disent un lézard,

Qui t'engendra d'une tigresse;

Autres, un dragon, d'une ânesse;

Un renard, d'un caméléon;

Un rhinocéros, d'un lion;

Un crocodile, d'une autruche ;

Un loup-cervier, d'une guenuche.

Pour moi je te mets au-delà

De tous ces vilains monstres-là. (p. 346-347)

- dans le cas du régime sérieux, on parle de transposition : Vendredi ou les limbes du Pacifique, constitue un bel exemple de transposition.

\section{Les principales formes de transformations}

Genette présente par le détail les différentes formes de transformations réalisées dans le cas de la transposition, qui lui apparaît comme la pratique hypertextuelle la plus courante et la plus importante. Impossible ici d'en présenter la somme, 
même en résumé. Je me limiterai donc à évoquer celles qui sont les plus fréquentes ou les plus significatives.

Ainsi, il peut s'agir de

- transformation quantitative, par réduction - Vendredi ou la vie sauvage compte 151 pages, contre 245 pour Vendredi ou les limbes du Pacifique -, ou par augmentation - Amélie Nothomb a publié en 2015 la réécriture d'une nouvelle d'Oscar Wilde, "Le crime de Lord Arthur Savile» sous la forme d'un roman, Le Crime du comte Neville;

- de mise en prose, voire de versification, comme c'est le cas lorsque le roman de Daniel Defoe a été réécrit par Saint-John Perse sous la forme du poème Images à Crusoé;

- de transvocalisation : alors que le roman de Defoe est tout entier écrit à la première personne, celui de Tournier fait alterner une narration à la troisième personne et une autre, à la première, celle du log-book; Vendredi ou la vie sauvage, lui, est entièrement narré à la troisième personne;

- de translation, temporelle - le Robinson de Tournier fait naufrage un siècle plus tard - , ou spatiale - le Pacifique de Tournier remplace l'Atlantique de Defoe;

- de transvalorisation: alors que, dans le roman de Defoe, c'est la civilisation qui l'emporte, Tournier fait au contraire de Vendredi l'éducateur de Robinson.

\section{Une écriture sous contraintes}

Même si elle ne figure pas parmi les multiples contraintes répertoriées par l'Oulipo, la réécriture mérite sans doute d'être 
considérée comme une forme d'écriture sous contraintes. Du reste, ce n'est sans doute pas un hasard si l'Oulipien Queneau a produit, dans ses Exercices de style, un bel exemple de réécriture, puisqu'il constitue à la fois la parodie d'un thème il s'agit de la même brève histoire — et du pastiche de 99 styles.

\section{Bibliographie}

ANGENOT, Marc. (1983), "L'intertextualité : enquête sur l'émergence et la diffusion d'un champ notionnel », Revue des sciences humaines, vol. LX, $\mathrm{n}^{\circ} 189$, janvier-mars, p. 121-135.

ANONYME. (1694), Recueil de pièces curieuses et nouvelles, t. 2, La Haye, Adrian Moetjens.

Bakhtine, Mikhaïl. ([1929] 1970), La Poétique de Dostoïevski, Paris, Seuil, coll. « Pierres vives ».

BARTHES, Roland. (1973), «Théorie du texte», dans Encyclopædia Universalis, vol. XV, p. 1015.

—. (1973), Le Plaisir du texte, Paris, Seuil, coll. « Tel Quel ».

BAYARD, Pierre. (2009), Le plagiat par anticipation, Paris, Minuit, coll. « Paradoxe».

Chamoiseau, Patrick. (2002), L'Empreinte à Crusoé, Paris, Gallimard.

DEFoE, Daniel. (1719), The Life and Strange Surprizing Adventures of Robinson Crusoe, of York, Mariner, Londres, W. Taylor.

Eco, Umberto. (1988), "Esquisse d'un nouveau chat», dans Pastiches et postiches, Paris, Messidor, p. 73-78.

EIGELDINGER, Marc. (1987), Mythologie et intertextualité, Genève, Slatkine. 
FERRON, Jacques. (1998), "Le petit chaperon rouge », dans Contes, Montréal, Les Presses de l'Université de Montréal, coll. « Bibliothèque du Nouveau Monde », p. 260-263.

GenetTe, Gérard. (1982), Palimpsestes. La littérature au second degré, Paris, Seuil, coll. « Poétique ».

JEnNy, Laurent. (1976), "La stratégie de la forme», Poétique, no 27, p. 257-281.

KrISTEVA, Julia. (1967), «Bakhtine: le mot, le dialogue et le roman », Critique, n 239, p. 44.

—. (1969), Sèméiôtikè. Recherches pour une sémanalyse, Paris, Seuil, coll. «Tel Quel».

MALLARMÉ, Stéphane. ([1897] 1974), "Crise de vers », dans CEuvres complètes, Henri Mondor et G. Jean-Aubry (éd.), Paris, Gallimard, coll. «Bibliothèque de La Pléiade», p. 360368.

Noтномв, Amélie. (2012), Barbe bleue, Paris, Albin Michel.

- . (2015), Le Crime du comte Neville, Paris, Albin Michel.

PIERSSENS, Michel. (1988), «Épistémocritique », Spirale, no 77, mars, p. 8.

Proust, Marcel. ([1920] 1984), «À propos du style de Flaubert », dans Contre Sainte-Beuve, précédé de Pastiches et mélanges et suivi de Essais et articles, Paris, Gallimard, coll. "Bibliothèque de La Pléiade », p. 586-600.

QUENEAU, Raymond. (1947), Exercices de style, Paris, Gallimard.

Rey-Debove, Josette et Alain Rey (dir.). (2004), Le nouveau Petit Robert, Paris, Dictionnaires Le Robert.

RIFFATERRE, Michael. (1979a), «Sémiotique intertextuelle : l'interprétant », Revue d'esthétique, n 1-2, p. 128-146.

-. (1979b), La Production du texte, Paris, Seuil, coll. «Poétique ». 
-. (1980), «La trace de l'intertexte», La Pensée, $\mathrm{n}^{\circ} 215$, octobre, p. 4-19.

RobBe-GriLlet, Alain. (1959), Dans le labyrinthe, Paris, Minuit.

SCARron, Paul. ([1648-1653] 1988), Le Virgile travesti, Paris, Classiques Garnier.

SOLLERS, Philippe. (1968), "Écriture et révolution», dans Tel Quel, Théorie d'ensemble, Paris, Seuil, coll. «Tel Quel », p. 6779.

Todorov, Tzvetan. (1965), Théorie de la littérature. Textes des formalistes russes, Paris, Seuil, coll. «Tel Quel».

Tournier, Michel. (1967), Vendredi ou les limbes du Pacifique, Paris, Gallimard.

—. (1971), Vendredi ou la vie sauvage, Paris, Gallimard.

-. (1978), "La fin de Robinson Crusoé», dans Le Coq de bruyère, Paris, Gallimard, p. 19-24

LE TRÉSOR DE LA LANGUE FRANÇAISE informatisé, $<$ http://atilf.atilf.fr/dendien/scripts/tlfiv4/showps.exe?p=c ombi.htm;java=no>.

WILDE, Oscar. (1891), Lord Arthur Savile's Crime and Other Stories, Londres, James R. Osgood, McIlvaine and Co.

WinCKLER, Martin. (2004), Les Trois Médecins, Paris, P.O. L. 